\title{
Ensuring Patient Safety and Access in Cancer Care
}

\author{
Katy Winckworth-Prejsnar, MPH ${ }^{\mathrm{a}}$; Elizabeth A. Nardi, MS ; James McCanney, JD; ; F. Marc Stewart, MD; \\ Terry Langbaum, MASc; Bruce J. Gould, MD; C. Lyn Fitzgerald, MJa; and Robert W. Carlson, MDa,e
}

\begin{abstract}
The inability to obtain the right high-quality cancer care in a timely and safe manner can have devastating results for patients. As cancer care becomes inundated with cutting edge and novel treatments, such as personalized medicine, oral chemotherapy, biosimilars, and immunotherapy, new safety challenges are emerging at increasing speed and complexity. Moreover, shifting federal healthcare policies could have significant implications for the safety and access to high-quality and effective cancer care for millions of patients with cancer. Challenges and opportunities in ensuring patient access to safe, affordable, and high-quality cancer care remain significant within the policy landscape. To address these concerns, NCCN hosted the Ensuring Safety and Access in Cancer Care Policy Summit in June 2017 to discuss pertinent patient safety issues and access implications under the Trump administration, as well as policy and advocacy strategies to address these gaps and build on opportunities moving forward.
\end{abstract}

J Natl Compr Canc Netw 2017;15(12):1460-1464 doi: 10.6004/jncen.2017.7049

\section{Executive Summary}

Patient safety is an integral component of high-quality and effective medical care. The stakes are especially high in oncology, where avoiding errors is imperative to delivering safe and effective radiation therapy, chemotherapy, and other high-risk treatments. Changing paradigms in cancer treatment, including oral chemotherapy, personalized medicine, biosimilars, and immunotherapy, create evolving safety challenges for the oncology community. Moreover, shifting federal healthcare policies could have significant implications for the safety and access to high-quality and effective cancer care for millions of patients with cancer. Challenges and opportunities in ensuring patient access to safe, affordable, and high-quality cancer care remain significant within the policy landscape.

To explore current patient safety and access issues in oncology, NCCN convened the NCCN Policy Summit: Ensuring Safety and Access in Cancer Care in Washington, DC, on June 15, 2017. Oncology stakeholders

From ${ }^{2}$ National Comprehensive Cancer Network, Fort Washington, Pennsylvania; ${ }^{b}$ Fred Hutchinson Cancer Research Center/Seattle Cancer Care Alliance, Seattle, Washington; 'The Johns Hopkins Hospital, Baltimore, Maryland; dNorthwest Georgia Oncology Centers, Marietta, Georgia; and 'Fox Chase Cancer Center, Philadelphia, Pennsylvania. Submitted October 5, 2017; accepted for publication October 9, 2017. (supplemental eAppendix 1, available with this article at JNCCN.org) gathered to discuss pertinent patient safety issues and access implications under the Trump administration, as well as policy and advocacy strategies to address these gaps and build on opportunities moving forward. The program consisted of presentations and 2 roundtable discussions with vigorous dialogue and audience comments and questions.

\section{Safety and Accountability in Cancer Care: Past, Present, and Future}

Patient safety issues within the United States healthcare system were brought to light in 1999 when the Institute of Medicine (IOM) released a groundbreaking report, "To Err is Human: Building a Safer Health System." The report highlighted critical patient safety issues through 2 studies that showed at least 44,000, and possibly as many as 98,000, people die in hospitals annually because of preventable medical errors. ${ }^{1}$ Most often, medical errors result from system design or system processes 
that either lead to mistakes or fail to prevent them. Rather than focusing on human errors, the IOM report emphasized the need for designing systems that prevent human errors and emphasize optimal patient safety. The report helped start a dialogue and led to patient safety initiatives at the organizational and national levels.

Organizations often use root cause analysis to analyze their errors and determine causes of problems, while trying to understand why; however, root cause analysis may not offer solutions on how to prevent future errors. ${ }^{2}$ Organizations find solutions such as technology changes and institutional-level modifications to be the most effective at preventing medical errors, whereas less effective and less sustainable solutions include counseling and disciplinary changes. ${ }^{3}$ Over time, an emphasis on system and cultural changes has led to the emergence of high-reliability organizations. Maintaining a balance between intentionally designed systems, professional accountability, and a culture of safety is what drives organizations to become and remain high-reliability organizations.

Various patient safety issues move beyond the institutional level and become broader national initiatives. Addressing safety issues throughout the cancer care continuum must also be met with an increased focus on guidelines, awareness, resources, and training. An NCCN Best Practices Committee survey identified catheter line infections and/or urinary tract infections, falls, patient care transitions, communication hand-off between care teams, and hand hygiene as the top pressing issues in patient safety at their institutions (Figure 1). ${ }^{4}$ Additionally, NCCN has conducted several alliance-wide safety initiatives. The NCCN Just Bag It! campaign promotes the delivery of vincristine diluted in a $50-\mathrm{mL}$ mini-intravenous drip bag rather than a syringe so that the drug can only be given intravenously and cannot be incorrectly and fatally injected into the spinal fluid. NCCN also promotes the use of autoinjector-delivered epinephrine at a fixed, safe dose for the treatment of allergic reactions to avoid confusion with epinephrine dosing and route of delivery for the treatment of cardiac arrest. ASCO's Quality Oncology Practice Initiative Certification Program evaluates oncology practice performance on certification standards that affect patient safety and care. ${ }^{5}$ Studies show that practices are often not fully compliant at the onset; however, the program promotes increased patient safety by identifying practice modifications that help achieve certification. ${ }^{6}$ These initiatives highlight the need for standards of auditing and measuring in order to continue to achieve safe practices.

Despite progress in the adoption of systems and initiatives to advance safe practices, patient safety gaps continue to exist, particularly in cancer care. Although clinical advances in cancer care are vital to patients, there are emerging risks associated with unknown long-term effects of novel cancer treatments. As cancer care becomes inundated with cutting edge and novel treatments, such as personalized medicine, oral chemotherapy, biosimilars, and immunotherapy, new safety challenges are emerging at increasing speed and complexity.

In the United States, approximately 3\% of people diagnosed with cancer participate in clinical trials. ${ }^{8}$ Low clinical trial participation can lead to unintended safety risks in cancer care, because information is focused on limited populations and shorter-term side effects. For example, an FDA analysis found that patients aged $>80$ years have a cancer incidence of $16 \%$, yet this population only makes up $4 \%$ of clinical trial participants. ${ }^{9}$ In order to ensure that new drugs are safe for all patient populations, proportional representation of each type of unique patient population is key.

Drug safety is monitored through pharmacovigilance, or the science related to the detection, assessment, understanding, and prevention of adverse effects or any other drug-related problem. ${ }^{10}$ Pharmacovigilance should occur throughout the life cycle of a drug, from development through postmarketing surveillance. Panelists noted that when pharmacovigilance is conceptualized, it is often from a drug perspective as opposed to a patient perspective. ${ }^{7}$ In other words, pharmacovigilance does not yet capture combined effects of drugs with radiation or drug regimens combined with other chemotherapeutic agents, as well as the short- or long-term combined drug toxicities for patients. The shift toward realworld data, however, is helping to monitor drugs longitudinally in real-world patient populations and is leading to smarter designed studies that capture a more accurate and concise picture of patient populations. Finally, electronic health record (EHR) programs coupled with technologically advanced interactive intelligence promises to improve safety and, at the same time, relieve physicians of many documen- 
Winckworth-Prejsnar et al

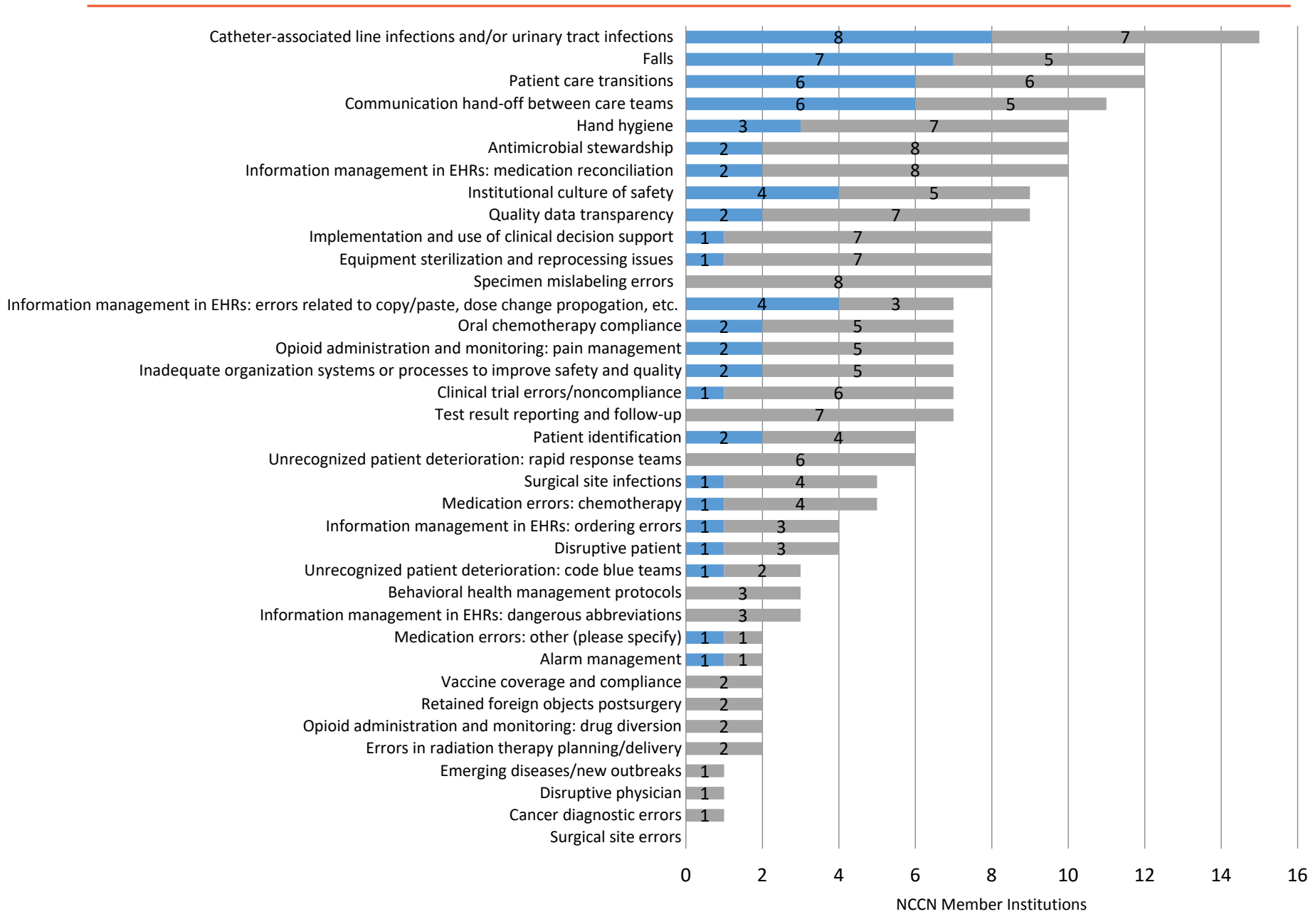

- Top 4 patient safety concerns

- Secondary concerns (devoting significant effort to)

Figure 1. NCCN Best Practices Committee survey: top patient safety concerns (NCCN Member Institutions, N=16).

Abbreviation: EHR, electronic medical record.

tation and ordering requirements, allowing them to focus on interaction with patients and making key, thoughtful healthcare decisions.

Real-world data and evidence allow for retrospective analysis and prospective evaluation, including the incorporation of claims data, cancer registries, and EHRs. ${ }^{7}$ Using EHRs as a data source allows for longitudinal data collection that represents the totality of a population and may capture patient experiences within the context of a single drug, combinations of drugs, or multiple treatments. More specifically, health information technology vendors are working with the FDA to explore analytic approaches, clinically relevant end points, and safety assessment methods using real-world evidence in order to provide new insights into the safety and ef- fectiveness of emerging anticancer therapies, such as immunotherapeutic agents. Ultimately, the increase in publicly available, digestible, and understandable data should improve cost-efficiency, clinical outcomes, and the patient experience.

As healthcare costs continue to increase, ${ }^{11}$ uninsured, underinsured, or insured customers with high deductible plans could bear more of the upfront drug cost burden, potentially limiting access or reducing adherence to life-saving drugs, ${ }^{12}$ which in turn creates safety issues when patients are not receiving appropriate therapies or dosing. The panel discussed the movement toward defining and delivering highquality cancer care through payment models based on value rather than volume. Integral components of the Patient Protection and Affordable Care Act 
(ACA), Medicare's Hospital Value-Based Purchasing (VBP) Program, and the Medicare Access and CHIP Reauthorization Act (MACRA) include a focus on improving patient safety and access to highquality care. The best way to stem the rising cost tide is to increase the use of value-based care decisionmaking tools. Additionally, benefit plans that incorporate value-based models are more likely to avoid overuse or misuse, therefore saving patients money. ${ }^{7}$

\section{Ensuring Access to High-Quality Cancer Care}

Access to appropriate and safe cancer care is of vital importance to all patients with cancer and their families. Proposed efforts to replace the ACA could alter the coverage and continuity of high-quality cancer care that many patients receive and expect; it could also place at risk provisions of the law that provide insurance protections from coverage denial for preexisting conditions or annual and lifetime caps. Alternatively, future policy changes could potentially address issues affecting the current model of individual exchange insurance markets established under the ACA, such as lower than expected enrollment, somewhat higher than expected risk enrollment, decreasing and geographically limited payer participation, as well as the high premiums and out-of-pocket costs (deductibles, copays, and coinsurance) for some patients currently covered under these exchange markets. In addition to increases in high deductible plans through the marketplace exchanges, high drug copays including specialty drugs, limited network of specialty cancer providers, and reimbursement based on sites of care could have severe implications for patient access, costs to the healthcare system, and overall quality of care for patients with cancer.

Since the passage of the ACA, 20 million people have gained insurance coverage, including those with cancer. ${ }^{13}$ In addition to coverage increases under the ACA, proven cancer screening and other preventive care are available at no cost for consumers resulting in increased utilization. For example, from 2011 to 2013, the increased screening rates under the ACA resulted in an $8 \%$ increase in the diagnoses of early-stage colorectal cancer among seniors aged $\geq 65$ years. ${ }^{14}$ At the time of writing, an estimated 2.3 million patients aged $<65$ years with cancer or a history of cancer rely on Medicaid and the Children's Health Insurance Program (CHIP) for their insurance, representing a $31 \%$ increase from 2013. ${ }^{15}$ Panelists raised concerns that the proposed reduction in Medicaid funding could leave the most vulnerable patient populations without affordable and timely access to optimal cancer diagnosis and evidence-based cancer treatment. ${ }^{16}$ Coverage losses estimated under previously proposed replacement plans could shift sites of care back to the emergency room, which panelists warned could have significant consequences for the quality and cost of cancer care. ${ }^{16}$

Although there were documented gains under the ACA, significant issues beset the individual exchange markets. The exchanges, established under the ACA, faced numerous hurdles since implementation, including changing regulations for insurers, lower than predicted enrollment, and higher risk enrollment. ${ }^{16}$ As a result, health plans participating in the exchange markets have seen higher risks and higher costs, with a significant number of insurance companies exiting the market. ${ }^{17}$ Moreover, under the ACA, an increasing number of health plans implemented "narrow networks" of providers as a cost containment strategy, leaving patients vulnerable to added financial and geographic burdens of out-of-network care. The lack of insurance providers available on some state exchanges combined with the proliferation of narrow networks created significant challenges for many patients to access and afford oncology care within a close vicinity. ${ }^{16}$ Optimally, patients should have access to provider networks that include experienced oncology experts and multidisciplinary cancer centers appropriate for their medical condition. In 2017, these fragile marketplaces experienced documented high-profile insurer exits, greater political uncertainty around market sustainability in the long term, and ambiguity around the future of cost-sharing reduction payments under the Trump administration. From a payer perspective, there was tremendous instability in the market exchanges from the onset, and there is a need for stability in the rules and regulations of the marketplaces moving forward. ${ }^{16}$

Beyond the instability of market exchanges under the ACA, panelists addressed concerns about access, affordability, and cost of care with shifts in sites of care from private community practices into hospitalowned facilities and practices, as well as the disputed role of the 340B Drug Discount Program. ${ }^{16}$ There is also mounting public concern that drug price increases could have severe implications for patient access and quality of care, ${ }^{8}$ with prescription drug spending 
Winckworth-Prejsnar et al

growing faster than any other health service recorded in 2015. ${ }^{18}$ Moving forward, panelists speculated the Trump administration will place more emphasis on outcomes-based contracts, FDA regulations to promote competition within the generic market, reforming the 340B Drug Discount Program, and Medicare Part B competition as strategies to address high drug pricing and overall healthcare costs. ${ }^{16}$

\section{Conclusions}

The inability to obtain the right high-quality cancer care in a timely and safe manner can have devastating results for patients-medically, psychologically, and financially. To ensure optimal safety in cancer care, the healthcare system needs to focus on continuity of care, develop systematic processes focused on safety, and establish systems designed to avoid adverse events. With the advent of molecular testing to determine targeted therapy for subsets of patients, more focus needs to be placed on the long-term safety, efficacy, access, and cost of potential targeted combination therapies. Moreover, as new safety challenges emerge at increasing speed and complexity, there is the prospect for using real-world data to better understand a myriad of patient safety issues, including patient experiences regarding use of a drug over time and the lateterm effects. Within the context of shifting healthcare policies, ultimately, health coverage alone does not provide access to timely, safe, affordable, high-quality care. As a community, it is important to articulate the importance of access to high-quality and affordable cancer care for all patients, quantify it, and then decide if there are tradeoffs as a community we are willing to make. Lastly, there is a consensus that moving toward reimbursement based on value, with a goal of quality improvement, is the direction the healthcare system in the United States needs to go. It will be important to track and measure the impact of such models relative to access, appropriate use of resources, safety, and ultimately outcomes for patients with cancer. The healthcare community needs to continue to engage all stakeholders in the value proposition and work together toward high-value cancer care for all patients.

\section{References}

1. Kohn LT, Corrigan JM, Donaldson MS, eds. To Err Is Human: Building a Safer Health System. Washington, DC: National Academy Press; 2000.

2. Kellogg KM, Hettinger Z, Shah M, et al. Our current approach to root cause analysis: is it contributing to our failure to improve patient safety? BMJ Qual Saf 2017;26:381-387.

3. Hettinger AZ, Fairbanks RJ, Hegde S, et al. An evidence-based toolkit for the development of effective and sustainable root cause analysis system safety solutions. J Healthc Risk Manag 2013;33:11-20.

4. Stewart FM. Patient safety and accountability. Presented at the NCCN Policy Summit: Ensuring Patient Access and Safety in Cancer Care; June 15, 2017; Washington, DC.

5. ASCO Institute for Quality. Available at: https://www.instituteforquality. org/. Accessed September 10, 2017.

6. Gilmore TR, Schulmeister L, Jacobson JO. Quality Oncology Practice Initiative Certification Program: measuring implementation of chemotherapy administration safety standards in the outpatient oncology setting. J Oncol Pract 2013;9(2 Suppl):14s-18s.

7. Safety and accountability in cancer care; past, present and future. Panel discussion at the NCCN Policy Summit: Ensuring Patient Safety and Access in Cancer Care; June 15, 2017; Washington DC.

8. Hudis C. Patient access to safe, high-quality cancer care under a new administration. Presented at the NCCN Policy Summit: Ensuring Patient Access and Safety in Cancer Care; June 15, 2017; Washington, DC.

9. Singh $\mathrm{H}$, Kanapuru B, Smith C, et al. FDA analysis of enrollment of older adults in clinical trials for cancer drug application: a 10-year experience by the U.S. Food and Drug Administration. Presented at the 2017 American Society of Clinical Oncology Annual Meeting; June 2-6, 2017; Chicago, IL.

10. Essential Medicines and Health Products: Pharmacovigilance. World Health Organization Web site. Available at: www.who.int/medicines/ areas/quality_safety/safety_efficacy/pharmvigi/en/. Accessed September 10, 2017.

11. Ramsey SD, Ganz PA, Shankaran V, et al. Addressing the American health-care cost crisis: role of the oncology community. J Natl Cancer Inst 2013;105:1777-1781.

12. Zafar SY, Peppercorn JM, Schrag D, et al. The financial toxicity of cancer treatment: a pilot study assessing out-of-pocket expenses and the insured cancer patient's experience. Oncologist 2013:18:381-390.

13. Health Insurance Coverage and the Affordable Care Act, 2010-2016. ASPE Web site. Available at: https://aspe.hhs.gov/pdf-report/health insurance-coverage-and-affordable-care-act-2010-2016. Accessed March $10,2017$.

14. Lissenden B, Yao NA. Affordable Care Act changes to Medicare led to increased diagnoses of early-stage colorectal cancer among seniors. Health Aff (Millwood) 2017;36:101-107.

15. Hearing to Consider the Graham-Cassidy-Heller-Johnson Proposal: before the United States Senate Committee on Finance, September 25, 2017 (statement of Dick Woodruff, American Cancer Society Cancer Action Network). Available at: https://www.acscan.org/statement-dick-woodruffgraham-cassidy-heller-johnson-proposal. Accessed November 28, 2019.

16. Barriers and opportunities in ensuring access to safe, high quality cancer care. Panel discussion at the NCCN Policy Summit: Ensuring Patient Safety and Access in Cancer Care; June 15, 2017; Washington DC.

17. Number of Issuers Participating in the Individual Health Insurance Marketplaces. The Henry J. Kaiser Family Foundation Web site. Available at: http://www.kff.org/other/state-indicator/number-of-issuersparticipating-in-the-individual-health-insurance-marketplace/?current Timeframe $=0 \&$ sortModel $=\% 7 \mathrm{~B} \% 22$ colId $\% 22: \% 22$ Location $\% 22, \% 22 \mathrm{~s}$ ort \%22:\%22asc\%22\%7D. Accessed September 5, 2017.

18. NHE Fact Sheet. Centers for Medicare \& Medicaid Services Web site. Available at: https://www.cms.gov/research-statistics-data-and-systems/ statistics-trends-and-reports/nationalhealthexpenddata/nhe-fact-sheet. html. Accessed September 5, 2017. 